lymphocyte structure looked entirely abnormal). This uneven development resulted at birth in absence or undetectable numbers of normally functioning cells of both $\mathrm{B}$ and $\mathrm{T}$ lines. Other children with SCID, at this point, would have been subjected to intense antigenic assault. Such assault could result in further damage to a lymphocyte population which was already functioning inadequately or perhaps not at all. In cases where the child is protected from such antigenic assault, it seems possible that

Copyright $\odot 1977$ International Pediatric Research Foundation, Inc. further opportunity for development may be provided, through increased survival time as well as through protection against overwhelming antigenic exposure. In the present case it appears that further development has already taken place; whether or not this will proceed to the emergence of an adequately functioning immune apparatus remains a question to be answered only by continued observation.

Pediat. Res. 11: 78-79 (1977)

\title{
V. Hematology
}

\author{
DONALD J. FERNBACH, KENNETH A. STARLING, AND JOHN M. FALLETTA
}

Department of Pediatrics, Baylor College of Medicine and the Research Hematology Laboratory, Texas Children's Hospital, Houston, Texas, USA

The hematologic surveillance of this patient revealed no dramatic differences from other children with SCID not maintained in a gnotobiotic environment. However, the absence of significant complicating infections - in fact, the absence of complications of any type-enabled this staff to accumulate data that probably reflect changes attributable to the natural history of this disorder more accurately than those that could be obtained under any other circumstnaces.

Details of the function of the neutrophils (Rebuck skin window studies and phagocytic studies) have been included in the section on immunology. No studies on the motility or phagocytic activity of the monocytes were done. The peripheral counts enumerated here were all obtained by conventional methods. Some of the early blood counts were done on blood obtained by heel puncture, but the majority were performed on venous blood samples.

\section{HEMOGLOBULIN, HEMATOCRIT, RED BLOOD CELL COUNT}

The lowest recorded hemoglobulin was $10.0 \mathrm{~g} / 100 \mathrm{ml}$, and the lowest hematocrit was $29.3 \%$ on the same day at approximately 3 months of age. During the first 3 months of life, the red cell count ranged from $3.51 \times 10^{6} / \mathrm{mm}^{3}$ to $4.44 \times 10^{6} / \mathrm{mm}^{3}$. All red cell counts thereafter have ranged from $4.0-5.0 \times 10^{8} / \mathrm{mm}^{3}$.

In consideration of the presumed role of enteric bacteria in absorption or metabolism of vitamin $\mathrm{B}_{12}(12)$, parenteral vitamin $\mathrm{B}_{12}, 500 \mu \mathrm{g}$, was administered monthly until May 1973 , at which time the patient was 18 months of age. Parenteral folic acid, 5 $\mathrm{mg}$, was also administered monthly until the same date. Up to that time, six bacterial organisms had penetrated the environment to colonize the child as shown in the section on gnotobiotic care. Only four of these persisted, although four other microbial organsims were later found to have colonized the child, so that by March 1974, there were eight persisting organisms. Except for the expected macrocytosis in the newborn period, there has been no alteration in the size of the peripheral red blood cells nor any other specific evidence of vitamin $B_{12}$ deficiency through August 1975.
It is appropriate to note at this point, relative to the role of bacterial organisms, that although no prothrombin times were recorded, only a few small ecchymoses were noted between 2 and 3 months of age. Vitamin $\mathrm{K}$ was administered parenterally until the spring of 1972 and orally until May 1973. No other bleeding manifestations have been documented and there is no reason to believe that there was significant hypoprothrombinemia at any time.

\section{LEUKOCYTES}

The absolute leukocyte levels are charted in Figure 1. The neutrophils, lymphocytes, and monocytes are similarly charted in Figure 2. Since September 1972 (age 1 year) the absolute leukocyte count has remained above $4.0 \times 10^{3} / \mathrm{mm}^{3}$. The last absolute lymphocyte count in excess of $2.0 \times 10^{3} / \mathrm{mm}^{3}$ was recorded on October 10,1972 when the child was 13 months of age. The absolute monocyte count gradually rose, with fluctuations, from 2.5 years of age to an apparently stable level at the present time. On a number of occasions the absolute monocyte count exceeded the absolute lymphocyte count. The total mononuclear cell count (monocytes plus lymphocytes) remained approximately 2,000 until September, 1974 when the patient was 3 years old. Since then, the combination of these counts has remained below $1,500 / \mathrm{mm}^{3}$. The bizarre appearance of the "monocyte" on the Rebuck skin window preparations (see Section IV) raises a question as to the exact nature of this cell which has a lymphocyte-like nucleus surrounded by an abundant polychromatophilic cytoplasm. In our experience, these are unlike mononuclear cells seen on other skin window preparations.

The single severe neutropenic episode during the second month of age occurred during gentamicin therapy which was administered in an attempt to eradicate the $S$. epidermidis. Within 3 days the count had risen to an adequate level although no changes were made in therapy and there was no apparent evidence of clinical infection at that time.

Eosinophil and basophil levels were unremarkable. During the first 3 months the eosinophils fluctuated up to an absolute count of $1,080 / \mathrm{mm}^{3}$ but since that time the level has ranged 
below $250 / \mathrm{mm}^{3}$. Basophils first appeared at 2 months of age and have persisted within the normal range thereafter.

In summary, changes observed in the various leukocyte levels have been consistent with earlier observations of children with this disorder (21).

\section{PLATELETS}

The platelet counts were normal to increased during the first month, declining during the second and third months of life to as low as $3,000 / \mathrm{mm}^{3}$. No petechiae were noted and no prolonged bleeding was observed in spite of numerous heel punctures. A return to normal occurring during the third month was followed by another episode of thrombocytopenia during the fourth month. Thrombocytopenia orginally occurred after discontinuation of colistin methate therapy and during the administration of gentamicin. The recurrence and recovery were observed during

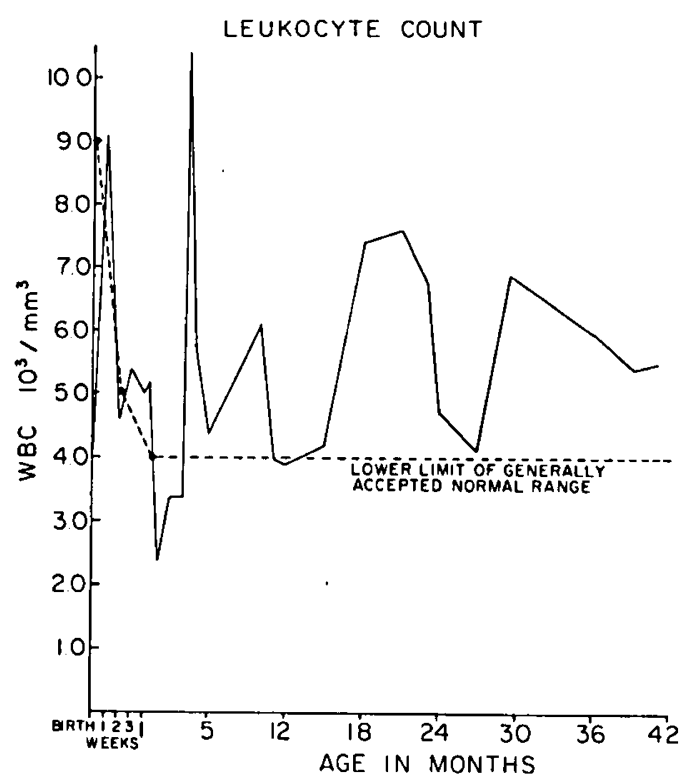

Fig. 1. Absolute leukocyte levels for severe combined immune deficiency patient to age 3.5 years. WBC: white blood cells.

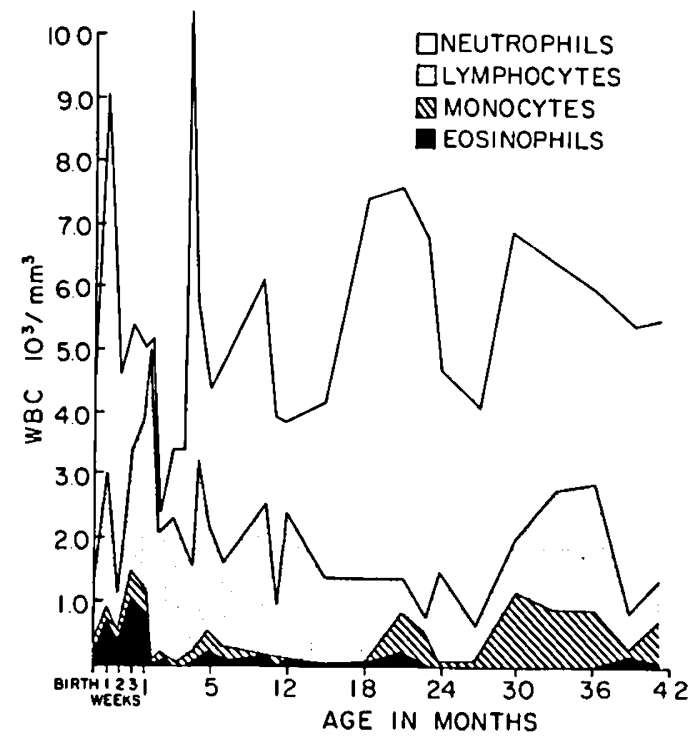

Fig. 2. Comparison of absolute levels of neutrophils, lymphocytes, monocytes, and eosinophils for severe combined immune deficiency patient to age 3.5 years. WBC: white blood cells.
Table 1. Bone marrow differential examination ${ }^{1}$

\begin{tabular}{l} 
Cell types \\
\hline Blasts \\
Promyelocytes \\
Neutrophilic myelo- \\
cytes \\
Eosinophilic myelo- \\
cytes \\
Neutrophilic meta- \\
myelocytes \\
Eosinophilic meta- \\
myelocytes
\end{tabular}

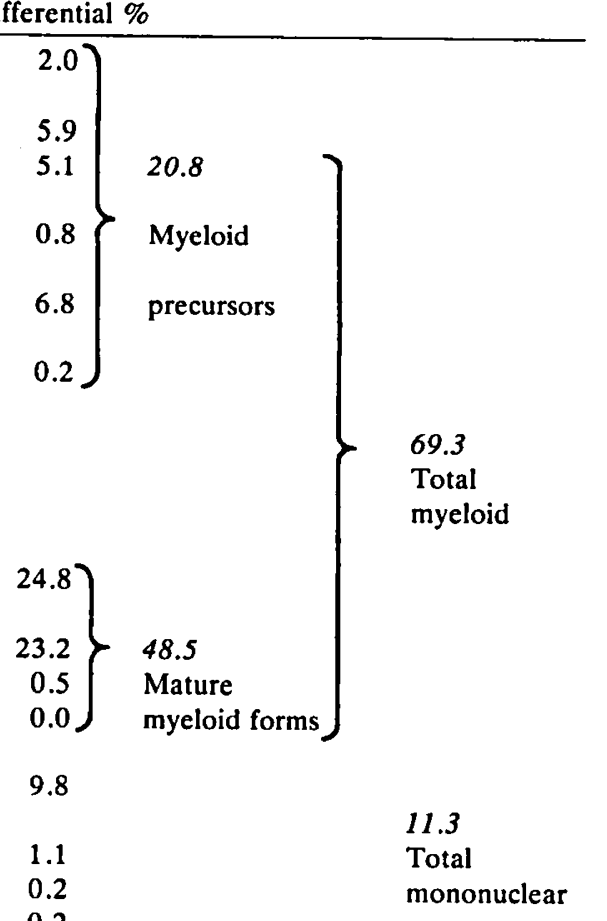

Neutrophils (poly-
morphonuclear)
Neutrophils (bands)
Eosinophils
Basophils

Lymphocytes

Atypical

Monocytes

Plasma cells

Reticulum cells

0.2

Proerythroblasts

Erythroblasts

Normoblasts

$\left.\begin{array}{r}0.4 \\ 12.5 \\ 6.5\end{array}\right\}$

19.4

Erythroid $\}$

precursors

Piatelets

Adequate

Megakaryocytes

Adequate

${ }^{1}$ Date obtained: May 19, 1975; age of patient: 44 months; no. of cells counted: 1,000 .

gentamicin therapy. During kanamycin therapy in the third month, platelet counts remained within the normal range.

At 1 year of age, 5 months after the last antibiotic treatment, another 3-month episode of thrombocytopenia was observed. This was detected 2 weeks after the second of three doses of transfer factor had been administered. The thrombocytopenia cleared up 3 months after the third dose of transfer factor and did not recur after a fourth dose was administered (5 months after the first administration).

No platelet antibody studies were done and no bone marrow specimens were obtained during the period of thrombocytopenia.

In summary, thrombocytopenia appears more likely to be related to the basic disease because occurrence and recovery did not seem to relate in a consistent pattern with antibiotic therapy, other therapy, or bacterial contamination of the environment.

\section{BONE MARROW}

The one bone marrow specimen was obtained from the posterior iliac crest at approximately 3.5 years of age. The results of the differential are presented in Table 1. Two plasma cells were seen, side by side. No abnormalities were seen in the myeloid or erythroid series and the M:E ratio (precursors only) of $1: 1$ was normal. Megakaryocytes and platelets were present in adequate numbers. Sequential bone marrow studies beginning in early life and at the time of peripheral blood changes would have been desirable, particularly with regard to the lymphocyte and plasma cell population and to thrombopoiesis. 